\title{
Fractures de la mandibule en pratique odontologique : à propos de 103 cas
}

\author{
Mandibular fractures in odontology: a report of 103 cases
}

SOUKÈYE DIA TINE', BABACAR TAMBA ${ }^{1}$, PAUL NIANG', CATHERINE GASSAMA BARRY', NDEYE FATOU KÉBÉ ${ }^{2}$, NDEYE FATOU GUĖYE², IBRAHIMA GUĖYE1', BOUBACAR DIALLO'

\begin{abstract}
RÉSUMÉ
La fracture mandibulaire constitue un motif fréquent de consultation en Odontologie à Dakar. Elle peut être considérée comme une urgence médicale de par l'hémorragie, mais aussi par les troubles respiratoires qui peuvent en découler.

Nous nous sommes fixés comme objectif d'étudier les aspects socio-démographiques, cliniques et thérapeutiques des fractures mandibulaires dans notre pratique.

Matériel et méthode : II s'agit d'une étude transversale et descriptive qui s'est déroulée de février 2003 à décembre 2006 et a concerné 103 patients ayant une fracture mandibulaire. Elle a eu pour cadre le service d'Odontologie de l'Hôpital général de Grand Yoff. Nous avons exploité le registre des patients et les fiches individuelles d'examen des patients

Résultats : L'âge moyen était de 27,6 ans avec des extrêmes allant de 3 ans et 73 ans. Le sex-ratio était de 3,9. Les délais de consultation varient de 0 à 40 jours. Le plus souvent les fractures mandibulaires sont dues à des accidents de la voie publique (46 cas, 44,70\%) et dans 57 cas (55,30 \%) la fracture siège dans la région symphysaire. Le traitement consiste à faire une prescription médicamenteuse avant tout acte, avec réduction suivie d'une contention orthopédique ou chirurgicale. (Med Buccale Chir Buccale 2009 ; 15: 137-145).
\end{abstract}

médecine buccale chirurgie buccale VOL. $15, \mathrm{~N}^{\circ} 3$ 2009 page 137

mots clés: fracture mandibulaire, aspect clinique, aspect thérapeutique

\section{SUMMARY}

The mandibular fracture constitutes a frequent motive for odontostomatology consultation at Darar. It can be considered as a medical urgency by the bleeding, but also respiratory disorders which can ensue from it.

We settled as objective to study the sociodemographic, clinical and therapeutic aspects of mandibular fractures in a dental consultation.

Material and method: It was a transverse and descriptive study which took place from february 2003 till december 2006 and concerned 103 patients victims of a mandibular fracture.

Results: The average age was of 27,6 years with extremes of 3 years and 73 years. The sex-ratio was 3,9. The periods of consultation vary from 0 to 40 days. The most frequent causes of mandibular fractures are the accidents of the public highway (46 cases, 44,70\%) and 57 fractures (55,30\% are localised in the symphyse. The treatment consists in making a medicinal prescription above all act, followed by a reduction and a contention, orthopaedic or surgical. (Med Buccale Chir Buccale 2009 ; 15: 137-145).

key words: mandibular fractures, clinical aspect, therapy aspect

1. Service de Chirurgie buccale Département d'Odontologie Université Cheikh Anta Diop Dakar

2. Service d'Odontologie Hôpital général de Grand Yoff Dakar

Demande de tirés à part:

Soukèye Dia Tine Chirurgie buccale Département d'Odontologie Université Cheikh Anta Diop BP 5005 Dakar diatine@ hotmail.com 
Les fractures mandibulaires se traduisent par une solution de continuité de l'os de la mandibule. Elles occupent une place importante dans les traumatismes maxillo-faciaux qui deviennent de plus en plus fréquents. Elles peuvent constituer une urgence médicale de par l'hémorragie ou la glossoptose qu'elles peuvent entraîner [1].

L'objectif de notre travail était d'étudier les aspects socio-démographiques, cliniques et thérapeutiques des fractures mandibulaires prises en charge dans le Service d'Odontologie de l'Hôpital général de Grand Yoff (HOGGY) entre février 2003 et décembre 2006.

\section{MATÉRIEL ET MÉTHODE}

II s'agit d'une étude transversale descriptive, concernant cent trois (103) patients victimes de fracture mandibulaire entre février 2003 et décembre 2006. Nous avons sélectionné tous les cas suivis dans le Service d'Odontologie et présentant une fracture de la mandibule durant notre période d'étude.

Les patients ont été pris en charge par quatre chirurgiens dentistes différents, assistés par un technicien supérieur en odontologie. Un chirurgien maxillo-facial vacataire est intervenu quelquefois à la demande des chirurgiens dentistes.

Nous avons exploité le registre et les fiches individuelles d'examen des patients.

L'âge, le sexe, le lieu de survenue et le type du traumatisme, la localisation du foyer de fracture, le délai de consultation, les moyens d'évacuation, les modalités thérapeutiques et les complications postopératoires sont les variables exploités.

Le logiciel SPSS 11.0 a été utilisé pour l'analyse des données.

Tableau 1 : Fréquence annuelle des fractures mandibulaires.

Annual frequency of the mandible fractures.

\begin{tabular}{|c|c|c|c|c|}
\hline Variables & $\mathbf{2 0 0 3}$ & $\mathbf{2 0 0 4}$ & $\mathbf{2 0 0 5}$ & $\mathbf{2 0 0 6}$ \\
\hline $\begin{array}{c}\text { Fréquence } \\
\text { absolue }\end{array}$ & 12 & 18 & 35 & 38 \\
\hline $\begin{array}{c}\text { Fréquence } \\
\text { relative (\%) }\end{array}$ & $11.64 \%$ & $17.47 \%$ & $34 \%$ & $36,89 \%$ \\
\hline
\end{tabular}

\section{RÉSULTATS}

\section{Aspects socio-démographiques Fréquence}

La fréquence par année des patients présentant une fracture de la mandibule est représentée sur le Tab. 1.

\section{Répartition selon l'âge}

Les limites d'âge se situent entre 3 et 73 ans. La moyenne est de 27,6 ans. La tranche d'âge la plus représentée est celle qui se situe entre 20 et 29 ans, qui représente $35 \%$ des patients reçus (Fig. 1).

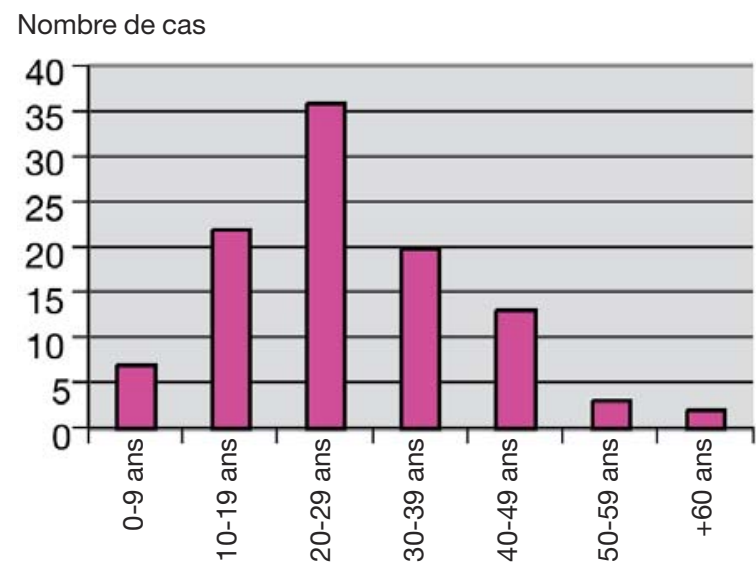

Figure 1 : Matériel de diagnostic disponible dans les services d'Odontologie.

Diagnostic equipment in dental teaching centers.

\section{Répartition selon le sexe}

Le sexe masculin est prédominant avec 82 patients $(79,60 \%)$ et 21 pour les femmes ce qui correspond à un sex-ratio de 3,9 (Fig. 2).

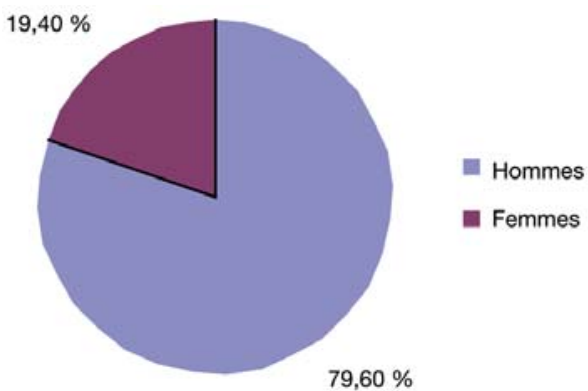

Figure 2 : Répartition des fractures mandibulaires selon le sexe.

Distribution of mandibular fractures according to the sex. 


\section{Répartition selon le lieu de résidence}

$81 \%$ des patients habitent dans la région de Dakar, $16 \%$ en dehors de Dakar et sa région et $3 \%$ n’habitent pas le Sénégal, et viennent essentiellement de la Guinée (Fig. 3).

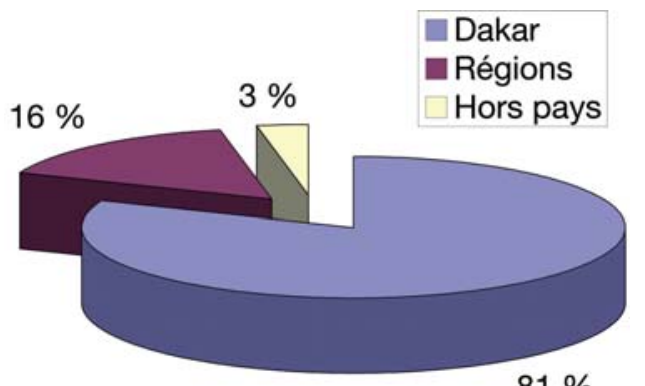

$81 \%$

Figure 3 : Répartition des fractures mandibulaires selon le lieu de résidence des victimes.

Place of mandibular fractures of victims residence.

\section{Répartition selon la profession}

Toutes les catégories socio-professionnelles sont représentées. Le groupe des ouvriers représente $25,25 \%$ des patients reçus, les écoliers 16,50 \% (étudiants et élèves), les sportifs $5 \%$ (lutteurs, footballeurs et pratiquants d'arts martiaux), les chauffeurs 5,8\% ; un nombre important de patients est sans profession (26,20\%).

\section{Répartition selon le délai de consultation}

Les délais de consultation varient de 0 à 40 jours. La moitié des patients est venue en consultation le jour même de la survenue du traumatisme $(50,50 \%), 10$ un jour après le traumatisme, 17 une semaine après le traumatisme $(16,50 \%)$ et six un mois après (Fig. 4).

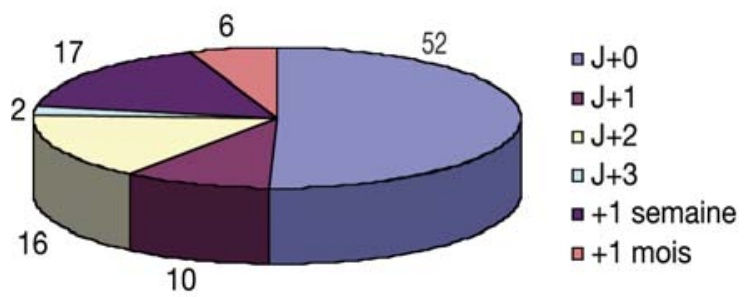

Figure 4 : Répartition des fractures mandibulaires selon le délai de consultation.

Distribution of mandibular fractures acccording to the delay of consultation.

\section{Lieu de survenue du traumatisme}

Une grande partie des traumatismes ont eu lieu à Dakar et dans sa banlieue : 76 cas soit $71,80 \%$. Les autres régions du Sénégal ont enregistré 24 cas (Thiès, Diourbel, Saint-Louis, Fatick et Matam). Trois cas $(2,90 \%)$ ont eu lieu hors du Sénégal : 2 cas en Guinée et un au Mali (Fig. 5).

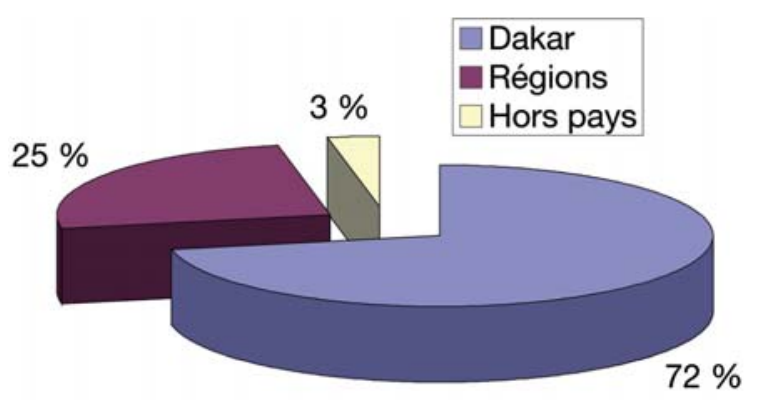

Figure 5 : Répartition des fractures mandibulaires selon le lieu de survenue du traumatisme.

Distribution of mandibular fractures according to the place where the traumatism occured.

\section{Répartition selon les moyens d'évacua- tion}

Les taxis et les ambulances (SUMA ou sapeurs pompiers) ont été les plus utilisés pour acheminer les malades, respectivement $35 \%$ et $32 \%$ des patients ont eu recours à ces moyens de transport, $11,80 \%$ à un véhicule personnel et 20,84 \% aux transports en commun pour se rendre à l'HOGGY. Chez 2,50\% des patients le moyen de transport utilisé n'a pas été précisé (Fig. 6).

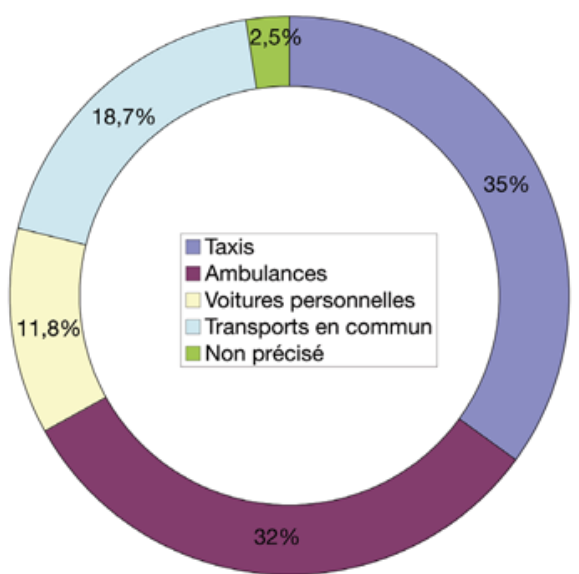

Figure 6 : Répartition des fractures mandibulaires selon le moyen d'évacuation.

Distribution of mandibular fractures according to the means of evacuation médecine buccale chirurgie buccale VOL. $15, \mathrm{~N}^{\circ} 3$ 2009 page 139 


\section{Aspects cliniques et radiologiques}

\section{Etiologies}

La cause la plus fréquente des fractures mandibulaires est représentée par les accidents de la voie publique (46 cas) et $87 \%$ par véhicule, viennent ensuite les chutes (16 cas) du haut d'un arbre, dans un escalier, dans la cour d'une maison ou d'une école, les rixes, les agressions et les accidents du travail ( 9 cas chacun) ; les coups de pied d'un animal domestique ou de trait (âne, cheval) (4 cas) ; la pratique de sports (football, lutte, arts martiaux) (7 cas). Dans ces 103 cas, on retrouve également une fracture balistique par grenade lacrymogène ; une fracture pathologique sur un carcinome mandibulaire et une fracture après extraction d'une dent de sagesse enclavée. Dans un dossier la cause n'était pas précisée (Tab. 2).

médecine buccale chirurgie buccale

page 140

\section{Bilan radiologique}

Le diagnostic a été confirmé par un examen radiologique : une incidence maxillaire défilé dans 71 cas et une incidence face basse pour 68 cas.
Une radiographie panoramique a été réalisée dans 18 cas et dans 13 cas une incidence de Blondeau, une incidence de Schüller dans 3 cas pour l'examen des ATM ; dans 2 cas, la radiographie rétroalvéolaire a permis de visualiser des foyers dentaires. Un scanner a été réalisé chez un polytraumatisé qui a présenté de multiples traits de fracture de la mandibule et une fracture du bras gauche.

\section{Localisation de la fracture}

La symphyse est le siège du foyer de fracture dans $55,30 \%$ des cas. 34 patients présentaient une double fracture avec un foyer dans la région de la symphyse associé à un autre dans l'angle ou la branche horizontale de la mandibule ; 61 patients un seul trait de fracture ; 4 patients trois traits et un patient plus de trois traits de fracture.

\section{Aspects thérapeutiques Traitement médicamenteux}

92,34 \% des patients ont reçu une prescription antibiotique à base d'amoxicilline ou de péni-

Tableau 2 : Répartition des fractures mandibulaires selon l'étiologie. Distribution according to the etiology.

\begin{tabular}{|l|c|c|}
\hline \multicolumn{1}{|c|}{ Variables } & Fréquence absolue & Fréquence relative (\%) \\
\hline Accidents voie publique & 46 & 44,6 \\
\hline Chutes & 16 & 8,7 \\
\hline Accidents travail & 9 & 8,7 \\
\hline Rixes & 9 & 8,7 \\
\hline Agressions & 9 & 3,8 \\
\hline Coups de pied d'animaux & 4 & 6,7 \\
\hline Sports & 7 & 2,9 \\
\hline Autres* & 3 & 0,9 \\
\hline Non précisé & 1 & 8,5 \\
\hline
\end{tabular}

* fracture balistique par grenade lacrymogène/ fracture pathologique suite à un carcinome mandibulaire/fracture iatrogène suite à l'extraction d'une dent de sagesse enclavée 
cilline $\mathrm{G}$, associée à un traitement antalgique à la demande chez $82,50 \%$ des patients : un traitement anti-inflammatoire a été prescrit dans $40 \%$ des cas. Seuls $1,90 \%$ des cas ont bénéficié d'une vaccination anti-tétanique. Un traitement traditionnel a été pratiqué par 5,80\% des patients.

\section{Traitement squelettique}

Le traitement orthodontique est assuré par la réduction et la contention des foyers de fracture puis par la rééducation. La réduction des foyers de fractures a été effectuée immédiatement par traction manuelle dans $90 \%$ des cas, et progressivement par traction à l'aide d'élastiques orthodontiques dans $9,70 \%$ des cas.

Ligatures et arcs ont permis d'assurer un blocage inter-maxillaire dans 71 cas, tandis que le cerclage péri-mandibulaire a été utilisé pour une contention monomaxillaire dans 14 cas. Les ligatures de Zylmer (Leblanc) ont été réalisées dans $35,90 \%$ des cas, suivis de celles d'lvy dans $22,30 \%$. Les arcs de Duclos ont été posés chez $26,20 \%$ des patients.

Le traitement chirurgical par ostéosynthèse a été réalisé chez cinq patients. L'abstention thérapeutique sous contrôle périodique a été adoptée chez trois patients alors que quatre patients ont été référés dans une autre structure sanitaire.

La durée de la contention a été en général de 45 jours chez les patients victimes de fracture de la branche horizontale. La fracture du condyle a nécessité une contention de 15 jours. Dans les cas présentant une complication (cellulite...) la durée de contention pouvait être prolongée jusqu'à 50 jours.

\section{Evolution}

Une guérison sans séquelles a été obtenue chez $91,4 \%$ des patients. Les complications observées ont été les suivantes: limitation de l'ouverture buccale $(6,80 \%$ des patients), cellulite $(3,80 \%)$, hypoesthésie transitoire $(2,90 \%)$, fracture non consolidée (1,90 \%). 3,80 \% des patients n'ont pas été revus et sur 2,90\% des dossiers, l'évolution n'a pas été précisée.

\section{DISCUSSION}

\section{Aspects socio-démographiques}

\section{Fréquence}

L'évolution de la fréquence des fractures de la mandibule dans notre consultation en odontologie pourrait s'expliquer par la recrudescence des traumatismes maxillo-faciaux au Sénégal qui serait liée :

- au non respect des règles de sécurité pour la circulation et pour le travail,

- à l'insécurité grandissante, dans certains milieux défavorisés où le chômage, l'alcoolisme et l'usage de drogue favorisant les rixes et les agressions. L'évolution des fractures mandibulaires dans notre étude diffère des résultats rapportés par Kontio et coll. ${ }^{[2]}$ en Finlande et Oikarinen et coll. ${ }^{[3]}$ au Canada. En effet dans ces pays développés, l'application d'une législation très rigoureuse a entraîné une baisse de la fréquence des fractures mandibulaires.

\section{Age}

Les jeunes adultes, âgés entre 20 et 29 ans, sont les plus représentés avec $35 \%$ des cas de fractures rencontrées. Ces résultats sont conformes avec ceux fournis par plusieurs auteurs notamment Sy [4], El Khatib et coll. [5], Dia [6], Kouakou et coll. [7] en Afrique et Alexander et coll. [8] au Canada. Les fractures mandibulaires sont fréquentes dans cette tranche d'âge du fait de la plus grande activité des victimes pendant cette période. Cette population s'adonne à la pratique de sport, et est très concernée par les rixes, les agressions et les prises de risque au volent lors de la conduite de voitures ou de motocycles.

\section{Sexe}

Nous avons noté la nette prédominance masculine dans notre étude avec un taux de presque $80 \%$ contre $20 \%$ pour les femmes soit un sexratio de quatre. Ceci est conforme avec les études effectuées par Sy [4], Dia [6], Alexander et coll. ${ }^{[8]}$ et Cantaloube et coll. ${ }^{[9]}$ qui ont rapporté des sexratio variant entre trois et cinq. Cette prédominance des hommes s'explique par le fait qu'ils sont plus orientés vers les activités physiques et médecine buccale chirurgie buccale VOL. $15, \mathrm{~N}^{\circ} 3$ 2009 page 141 
médecine professionnelles à risque de traumatismes. Les femmes sont plus exposées aux chutes et glissades lors des travaux domestiques.

\section{Lieu de résidence}

La grande majorité des patients réside dans la région de Dakar (81\%). En effet la capitale concentre la plus grande partie des activités socioprofessionnelles avec un trafic automobile très dense. II faut noter que $25 \%$ des fractures sont survenus dans les régions hors de Dakar, alors que seulement $16 \%$ des patients déclarent résider dans ces régions. Ainsi ils sont obligés de quitter leur localité pour venir se faire traiter à Dakar, la capitale dans des conditions de transport et d'existence très difficiles pour un blessé. Dans les régions, les étiologies les plus fréquentes sont la chute du haut d'un arbre ou d'un animal, les coups de pied d'animaux ; les accidents de la circulation concernent seulement $15,80 \%$ des cas. $3 \%$ des patients viennent de pays limitrophes (Guinée, Mali) au Sénégal en raison du manque de spécialiste ou d'un plateau technique approprié dans ces pays.

\section{Profession}

Dans notre étude, écoliers, ouvriers et chômeurs sont les plus représentés comme il a été rapporté dans l'étude de Cantaloube et coll. [9] effectuée au Sénégal. Dans notre pays les chômeurs et les travailleurs du secteur tertiaire y sont très représentés d'où leur proportion importante dans les cas de fractures.

Par contre les études de Kontio et coll. [2] en Finlande, et Oikarinen et coll. ${ }^{[3]}$ au Canada montrent que les sportifs sont plus victimes de fractures mandibulaires.

\section{Délai de consultation}

Les délais de consultation varient entre 0 et 30 jours, avec une moyenne de 3 jours. Près de $76 \%$ des patients ont consulté dans les 48 heures suivant le traumatisme. Cette consultation relativement tardive a été retrouvée dans l'étude de Soriano et coll. [10] en France, qui rapporte un délai moyen de deux jours, et dans d'autres réalisées au Sénégal $[4,6,9,13]$. Le retard de consultation dans notre série pourrait être expliqué par le faible niveau socio-économique de nos patients qui sont souvent confrontés à des difficultés financières, par la symptomatologie parfois frustre pouvant accompagner les fractures mandibulaires et aussi par le manque de spécialistes.

\section{Moyens d'évacuation}

$35 \%$ des traumatisés ont été évacués par taxi ou voiture particulière. Ces moyens de transport se trouvent être inadaptés au transport de blessés. Il est à déplorer l'absence d'un système de transport pour les urgences organisé et facilement accessible aux blessés.

\section{Aspects cliniques et radiologiques Etiologies}

L'étiologie la plus fréquente est constituée par l'accident de la voie publique ( $45 \%$ des cas dont la majorité par accident de voitures). Aucune des victimes ne portaient la ceinture ou le casque de sécurité. La conduite en état d'ivresse, la négligence ou l'excès de vitesse sont les principales causes des accidents comme il l'a été noté dans l'étude de Sy ${ }^{[4]}$. Dans les pays développés les accidents de la voie publique entraînent moins de traumatismes mandibulaires car le port de ceinture et de casque y est obligatoire et respecté, comme le constatent Rocton et coll. ${ }^{[12]}$ en France. Les chutes représentent $15,50 \%$ des étiologies. Celles du haut d'un arbre constitue une particularité sénégalaise se produisant généralement en milieu rural durant la période de la cueillette des fruits. La plupart des victimes sont des enfants comme le rapporte les études de Badiane ${ }^{[13]}$ et Larroque et coll. [11].

La recrudescence de la violence constatée dans notre pays pourrait être expliquée par l'usage de l'alcool, de la drogue et le chômage. Si dans notre étude cette violence ne représente que $15 \%$ des étiologies, dans l'étude de Rocton et coll. [12] en France elle vient en première position avec $57 \%$ des cas.

La pratique de sports est une étiologie non négligeable avec $7 \%$ des cas alors que dans l'étude de Sy ${ }^{[4]}$ les traumatismes par pratique du sport représentent $3,70 \%$ des cas. Selon Onyeaso et coll. ${ }^{[14]}$ au Nigeria, l'utilisation de gouttières de protection des arcades dentaires lors de la pratique sportive a participé à une baisse considérable du 
taux de traumatisés du massif facial. Ainsi son utilisation au Sénégal dans les sports violents tels que la lutte avec frappe doit être recommandée. Les accidents du travail comme il est rapporté dans l'étude de Sy ${ }^{4]}$ constituent la troisième cause des fractures mandibulaires $(8,70 \%$ des cas). Ils sont souvent le fait de la négligence, de l'insécurité, des horaires et des conditions de travail difficiles dans nos pays sous développés. Par contre Von Hove et coll. [15], dans une étude effectuée en France, rapporte un taux plus faible (3,70 \% des cas) qui s'explique par une législation plus rigoureuse avec l'application du code du travail dans les pays européens.

Les coups de pied d'animaux avec un taux de $4 \%$ représentent aussi une étiologie particulière des fractures mandibulaires au Sénégal. Elles se passent essentiellement dans les villages où l'élevage constitue une activité importante. Les enfants qui participent à l'entretien du bétail en sont les premières victimes comme le rapportent les différentes études effectuées au Sénégal par Sy ${ }^{[4]}$, Larroque et coll. ${ }^{[11]}$ et Badiane ${ }^{[13]}$.

\section{Bilan radiologique}

Les incidences face basse et maxillaire défilé ont été les radiographies de base dans notre étude, elles doivent être associées pour bien objectiver le foyer de fracture en vue de face et de profil. Elles ont permis de mettre en évidence la plupart des foyers de fractures et de faire le contrôle après traitement. Leur prédominance dans notre série s'explique par leur accessibilité à l'HOGGY, leur facilité d'exécution et leur coût peu onéreux. L'orthopantomogramme, qui a permis le diagnostic dans $17,50 \%$ des cas, est une incidence qui n'est pas toujours disponible dans notre pratique, en effet elle n'est pas réalisable à l'HOGGY. L'incidence de Schüller utilisée à trois reprises $(2,90 \%)$ a permis la mise en évidence des atteintes articulaires et des condyles mandibulaires. Dans 12,60 \% des cas l'incidence de Blondeau a permis de détecter les fractures du maxillaire associées à la fracture mandibulaire.

\section{Localisation de la fracture}

$55,30 \%$ des fractures de la mandibule sont localisées dans la région symphysaire. Ainsi la por- tion dentée constitue la partie la plus touchée par les fractures mandibulaires. En effet, de par sa position proéminente, elle constitue le pare-choc du visage comme cela a été noté dans l'étude de Sy ${ }^{[4]}$. Les fractures de l'angle, rarement isolées, viennent en 3ème position avec 16,50 \%. L'étude de Rocton et coll. [12], rapporte $32 \%$ de cas de fractures de l'angle associée à une fracture du condyle. Selon Fuselier et coll. [16], le risque de fracture mandibulaire touchant l'angle augmente de deux à trois fois lorsqu'il y a d'une dent de sagesse enclavée ou incluse. Ces résultats sont conformes avec ceux d'El Khatib et coll. [5].

Le plus souvent (60\% des cas) il s'agit d'une fracture unifocale. Badiane [13] dans une étude réalisée à Dakar, retrouve $70,30 \%$ de fracture unifocale. Pour Kouakou [7], en Côte d'Ivoire, ce type de fracture représente $62,50 \%$ des cas avec une prédominance de foyers situés dans l'angle mandibulaire. La fracture bifocale constitue $33 \%$ des fractures mandibulaires regroupant plus fréquemment les associations suivantes : symphyseangle ou symphyse-condyle, branche horizontalecondyle, branche verticale-condyle. Selon Gola et Cheynet [17], lorsque le point d'impact est sur le menton, la fracture survient d'abord dans la région de la symphyse puis la brusque rétropulsion de la mandibule poussant les condyles dans la cavité glénoïde aboutit à une fracture condylienne. Lorsqu'il y a un choc latéral au niveau de l'angle, la mandibule tend à se fermer, entraînant le plus souvent une fracture de l'angle couplée à une fracture symphysaire [1].

\section{Aspects thérapeutiques}

\section{Traitement médicamenteux}

L'antibiothérapie a été systématique avant tout acte thérapeutique car toute fracture intéressant la portion dentée mandibulaire est considérée comme une fracture ouverte. Aussi le risque infectieux est très important chez nos patients qui présentent une hygiène bucco-dentaire en général défectueuse. Cette prescription a été accompagnée d'un antalgique à la demande et d'un antiseptique local sous forme de bains de bouche. Les anti-inflammatoires ont été prescrits dans $40 \%$ des cas pour juguler un œdème post-traumatique. médecine buccale chirurgie buccale VOL. $15, \mathrm{~N}^{\circ} 3$ 2009 page 143 
médecine

\section{Traitement squelettique}

Il comprend la réduction, la contention et la rééducation.

La réduction peut se faire manuellement : elle consiste à reconstituer l'articulé dentaire en rapprochant les fragments fracturés par une traction mécanique manuelle. Elle précède tout acte de contention. Si cette traction manuelle ne suffit pas à rétablir l'articulé dentaire, la réduction se fera alors progressivement avec des fils élastiques orthodontiques. Cette réduction a été utilisée chez $10 \%$ de nos patients.

La contention fait appel à plusieurs méthodes. La méthode orthopédique est le type de contention le plus utilisé. On a surtout recours aux ligatures de type Zylmer ou Leblanc (36 \%) et à celles d'Ivy $(22,50 \%)$ comme rapporté dans l'étude effectuée par Sy [4]. Dans certains cas où la ligature est impossible, la pose d'arcs devient la méthode orthopédique la plus adaptée. L'arc de Duclos a été utilisé chez $26 \%$ des patients. Elle est facile à réaliser par des odontologistes et donne des résultats semblables à ceux de la pose de ligature.

La forte prédominance du traitement orthopédique s'explique par son coût relativement faible, accessible aux patients, par la consultation parfois tardive ne permettant plus un traitement chirurgical, ou par un plateau technique insuffisant par défaut de matériel chirurgical. Elle représente donc une méthode de traitement fiable, adaptée à notre pays où les ressources sont limitées et les chirurgiens maxillo-faciaux en nombre très insuffisant.

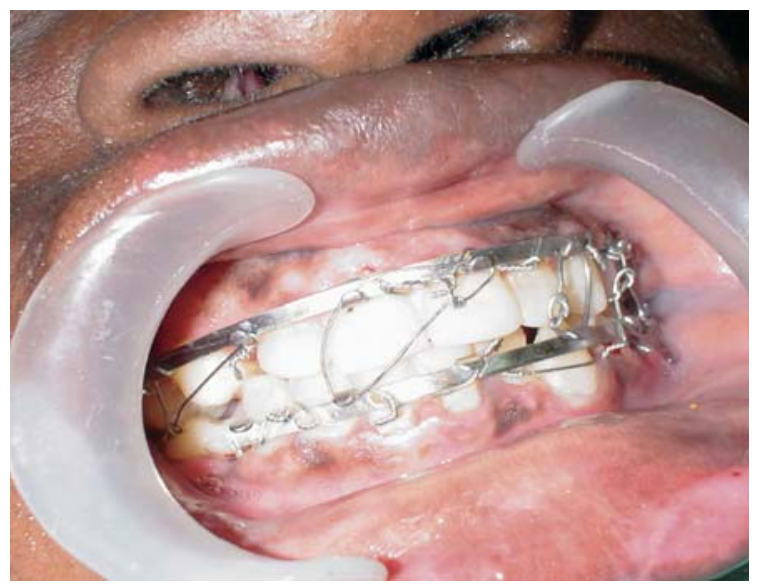

Figure 7 : Blocage inter-maxillaire par arcs et ligatures. Blocking maxillary by bows and ligatures.
Le traitement orthopédique donne des résultats presque similaires à ceux de la méthode chirurgicale comme l'ont montré Dia ${ }^{[6]}$ dans son étude réalisée à Dakar, et Ngouoni et coll. [18].

Le cerclage périmandibulaire a été utilisé dans $13,60 \%$ des cas. Cette technique est préconisée lorsque le blocage maxillo-mandibulaire est contre-indiqué. Elle est particulièrement utilisée chez la personne âgée, l'édenté total et l'enfant comme l'ont montré Ouedraogo et coll [19] dans son étude faite au Burkina Faso.

La contention de choix de la fracture mandibulaire prise en charge précocement se fait par ostéosynthèse aux fils d'acier ou par mini-plaques vissées. Cette méthode chirurgicale a été utilisée seulement dans $6 \%$ des cas. Par contre dans l'étude de Rocton et coll. [12], en France, $73 \%$ des patients ont bénéficié de l'ostéosynthèse de même dans celle de Soriano ${ }^{[10]}$ où la presque totalité des patients ont bénéficié d'une ostéosynthèse. On peut atteindre de tels pourcentages dans les pays développés car on dispose d'un plateau technique très relevé, de spécialistes et de structures spécialisées en nombre suffisant.

L'abstention thérapeutique est préconisée dans les cas de fractures incomplètes sans déplacement où l'articulé dentaire est conservé. Un de nos patients en a bénéficié. Elle doit être associée à une alimentation liquide ou semi-liquide et des contrôles réguliers.

La rééducation se fait par mécanothérapie à l'aide de l'exerciseur de Durcissac. Dans notre série par défaut de cet instrument, la " pince à linge » est utilisée, associée à la mastication de chewing-gum pour assurer la rééducation. Elle est recommandée aux patients ayant une limitation de l'ouverture buccale après dépose de la contention.

\section{Evolution}

Elle a été favorable dans $77,50 \%$ des cas. Cependant nous avons constaté quelques complications à type de limitation de l'ouverture buccale, de cellulite, ou de retard de consolidation, comme le rapportent Soriano et coll [10]. Alexander et coll. [8], en France, observent 5,30 \% de complications dont la moitié est d'ordre infectieux. Dans notre étude la complication infectieuse a été favorisée par une mauvaise hygiène bucco- 
dentaire ou un non respect de la prescription médicamenteuse.

\section{CONCLUSION}

La fracture mandibulaire constitue un motif fréquent de consultation en pratique odontologique dans un milieu hospitalier. Elle prédomine chez le jeune adulte entre 20 et 30 ans et de sexe masculin. La fréquence des fractures de la mandibule dans notre consultation pourrait s'expliquer par la recrudescence des accidents et de l'insécurité au Sénégal. La fracture sympysaire est la forme clinique la plus représentée dans notre étude.

\section{RÉFÉRENCES}

1 - Piette E, Reychler H. Traité de pathologies buccales et maxillo-faciales (pp 1483-1509). De Boeck Université Bruxelles, 1991.

2 - Kontio R, Surmen R, Ponkkonen H, Lindquist C, Laine P. Have the causes of maxillo-facial fractures changed over the last 16 years in Finland? An epidemiological study of 725 fractures. Dent Trauma $2005 ; 21$ : 14-9.

3 - Oikarinen K, Schutz P, Thalib L, Sandor GKB, Clokie G. Differences in the etiology of mandibular fractures in Kuwait,Canada and Finland. Dent Trauma 2004 ; 20 : 241-5.

4 - Sy A. Aspects cliniques et thérapeutiques des fractures mandibulaires. A propos de 53 cas colligés à l'Hôpital général de Grand-Yoff (HOGGY). Thèse de Chirurgie dentaire $n^{\circ}$ 21, Dakar, 2004.

5 - El Khatib K, Gradel J, Danino A, Mouaffak M, Malka G. Alimentation entérale par sonde naso-gastrique : intérêt après ostéosynthèse des fractures mandibulaires. Rev Stomatol Chir Maxillofac 2005 ; 106 : 13-5.

6 - Dia M. Les fractures de la mandibule. A propos de 54 cas observés colligés au niveau du service de Stomatologie du CHU Aristide Le Dantec. Thèse de Chirurgie dentaire $n^{\circ} 2$, Dakar, 1999.

7 - Kouakou NC, Bamba A, N'Cho KJC, Amicha-Alloh YC. Les fractures mandibulaires colligées au centre de consultations et de traitements odonto-stomatologiques (CCTOS) d'Abidjan. A propos de 24 cas. Rev Col Odontostomatol Afr Chir Maxillofac 2003 ; 10 : 18-21.

8 - Alexander JS, Meisami T, Georges K, Sandor B, Cameron MLC. Epidémiologie des fractures mandibulaires traitées à l'Hôpital général de Toronto : revue sur 246 cas. J Assoc Dent Can 2001 ; 67 : 640-4.

9 - Cantaloube D, Sans H, Larroque G, Le Bourgeois M. Les fractures mandibulaires. Considérations épidémiologiques et thérapeutiques. A propos d'une série récente de 100 cas observés à l'Hôpital principal de Dakar. Med Afr Noire 1987 ; 34 : 447-53.
La forte prédominance du traitement orthopédique s'explique par :

- un coût relativement faible,

- une consultation souvent tardive ne permettant plus un traitement chirurgical,

- un défaut de disponibilité du matériel chirurgical, - une facilité de réalisation par des odontologistes. Le traitement orthopédique donne des résultats presque similaires à ceux du traitement chirurgical. II représente donc une méthode thérapeutique fiable, adaptée à notre pays où les ressources sont limitées et les chirurgiens maxillofaciaux en nombre très insuffisant.

10 - Soriano E, Kankou V, Morand B, Sadek H, Raphael B, Bettega G. Fracture de l'angle mandibulaire : facteurs prédictifs des complications infectieuses. Rev Stomatol Chir Maxillofac 2005 ; 106 : 146-8.

11 - Larroque G, Jouen F, Cantaloube D, De Carvalho P, Younes $Y$. Epidémiologie des traumatismes maxillofaciaux au Sénégal. Rev Odontol Tropicale 1998 ; 10 : 194-8.

12 - Rocton S, Chaine A, Ernenwein D, Bertolus C, Rigolet A, Bertrand JC, Ruhin B. Fracture de la mandibule : épidémiologie, prise en charge thérapeutique et complication d'une série de 563 cas. Rev Stomatol Chir Maxillofac 2007 ; $108: 3-12$.

13 - Badiane MB. Les traumatismes maxillo-dentaires au Sénégal. Thèse de Chirurgie dentaire n 7, Dakar, 1989.

14 - Onyeaso C, Arowojolu MO, Okoje VN. Nigerian dentist's knowledge and attitudes towards mouthguard protection. Dent Trauma 2004 ; 20 : 187-91.

15 - Von Hove A, Lolom H, Sapanet M, Descrozailles JM. Les fractures mandibulaires. Etudes rétrospectives de l'activité du service de Chirurgie maxillo-faciale et Stomatologie du CHU de Poitiers de 1978 à 1997. Rev Stomatol Chir Maxillofac 2000 ; 101 : 309-18.

16 - Fuselier JC, Ellis E. 3rd, Dodson B. Do mandibular third molars alter the risk of angle fracture? J Oral Maxillofac Surg $2002 ; 60: 514-8$.

17 - Gola R, Cheynet F. Fracture de la mandibule. Encyc Odontostomatol 22-070-A-10. Elsevier, Paris, 1994.

18 - Ngouoni BG, Mathey-Manza, Moyikoua. Résultats du traitement des fractures mandibulaires. A propos de 169 cas. Med Afr Noire 1996 ; 43 : 529-32.

19 - Ouedraogo D, Konsem T, Gare JV, Beogo R, Ouoba K. Les fractures mandibulaires de l'enfant. A propos de 20 cas. Rev OdontoStomatol Trop 2006 ; 114 : 5-8. médecine buccale chirurgie buccale VOL. $15, \mathrm{~N}^{\circ} 3$ 2009 page 145 